\title{
Adoção do Sistema Eletrônico de Informações (SEI) pelo Ministério da Justiça: uma avaliação na ótica de usuários
}

\author{
Denise de Carvalho Pires Fulton \\ Universidade de Brasília (UnB) \\ Josivania Silva Farias \\ Universidade de Brasília (UnB) \\ Solange Alfinito \\ Universidade de Brasilia (UnB)
}

Juliana Pascualote Lemos de Almeida

Universidade de Brasília (UnB)

Este estudo investiga a atitude de usuários em relação ao Sistema Eletrônico de Informações (SEI), adotado pelo Ministério da Justiça (MJ) do Brasil, baseando-se em dimensões de adoção de tecnologia segundo Moore e Benbasat (1991). Aplicaramse questionários entre usuários do SEI $(\mathrm{N}=208)$, incluindo a verificação de evidências de validade da escala por análise fatorial exploratória. Como resultados, verificou-se que: há confiabilidade dos fatores e os itens das dimensões de vantagem relativa e compatibilidade compuseram um só fator; há atitude favorável ao SEl; grupos de usuários que utilizam o SEI há mais tempo diferiram em relação aos iniciantes quanto a vantagem relativa, compatibilidade e testabilidade do sistema; pessoas mais velhas valorizaram facilidade de uso e testabilidade, enquanto as mais jovens valorizam demonstrabilidade de resultados. O estudo recomenda reflexões sobre a variável vantagem relativa, por ter incorporado outro fator, e também sobre novas aplicações do instrumento em outros órgãos públicos adotantes do SEl.

Palavras-chave: avaliação tecnológica - usuário, tecnologia da informação, modernização administrativa, administração pública - estudo de caso

[Artigo recebido em 30 de novembro de 2016. Aprovado em 15 de agosto de 2018.] 
Adopción del Sistema de Información Electrónica (SEI) por el Ministerio de Justicia: una evaluación desde el punto de vista de los usuarios

Este estudio investiga la actitud de los usuarios en relación con el Sistema Electrónico de Información (SEI), aprobado por el Ministerio de Justicia (MJ) de Brasil, basado en la tecnología de las dimensiones de adopción de acuerdo con Moore y Benbasat (1991). Fueron aplicados cuestionarios entre los usuarios de SEI $(N=208)$, incluyendo la verificación de validez de la escala mediante análisis factorial exploratorio. Como resultado, se encontró que: existe confiabilidad de las mediciones y los elementos de las dimensiones de ventaja relativa y compatibilidad se incluyeron un solo factor; hay actitud favorable a SEl; grupos de usuarios utilizando el SEI hace más tiempo diferían en relación a los principiantes en ventaja relativa, compatibilidad y capacidad de prueba del sistema; las personas mayores aprecian la facilidad de uso y la capacidad de prueba, mientras que los más jóvenes aprecian la demostrabilidad de resultados. El estudio recomienda reflexiones sobre las variables de ventaja relativa, mediante la incorporación de otro factor, y también sobre nuevas aplicaciones del instrumento en otros organismos públicos adoptantes del SEl.

Palabras clave: evaluación tecnológica - usuario, tecnología de la información, modernización administrativa, administración pública - estudio de caso

\section{Adoption of Electronic Information System (SEI) by the Ministry of Justice: An assessment in the user perspective}

This study investigate the attitude of users regarding the Electronic Information System (SEI), adopted by the Ministry of Justice (MJ) of Brazil, based on technology adoption dimensions according to Moore and Benbasat (1991). Questionnaires were applied among users of SEI ( $\mathrm{N}=208)$, including verification of scale validity evidences by exploratory factor analysis. The results include: the reliability of the factors and the items of the dimensions of relative advantage and compatibility merged in only one factor; there is favorable attitude toward the SEl; user groups using the SEI for a longer period differ in relation to beginners, regarding relative advantage, compatibility and trialability of the system; older people valued ease of use and trialability, while younger ones valued result demonstrability. The study recommends reflections concerning to relative advantage variable, which incorporated other factor, and also indicates new applications of the instrument in other public organizations that adopt SEI.

Keywords: technology assessment - user, information technology, administrative modernization, public administration - case study 


\section{Introdução}

A inserção de novos sistemas no ambiente organizacional representa mudança de rotinas e objetiva tornar a execução dos processos de trabalho mais eficaz, além de facilitar a interface dos atores envolvidos (KLERING; ANDRADE, 2006). Abrange um aspecto importante de inovação e adoção de tecnologia, como aponta Rogers (1983), com sua Teoria da Difusão da Inovação (TDI), que instrui que a adoção e a difusão de inovações sejam motivadas pelo aumento da eficiência e desempenho organizacional, também conhecida como perspectiva de escolha estratégica (Dos SANTOS, 2007).

Pela TDI, o processo decisório de inovação se dá por seis estágios: condições prévias; conhecimento; persuasão; decisão; implementação; e confirmação. Especialmente na fase da persuasão, alguns atributos inerentes a uma inovação tendem a facilitar a sua difusão, a saber: vantagem relativa; compatibilidade; complexidade; testabilidade; e observabilidade (ROGERS, 1983).

Moore e Benbasat (1991), a partir dos atributos de Rogers (1983), consideram cinco dimensões avaliativas da adoção de tecnologia: vantagem relativa; compatibilidade; facilidade de uso; testabilidade; e demonstrabilidade dos resultados. Esses atributos são o foco de estudo desta pesquisa no que se refere ao Sistema Eletrônico de Informações (SEI), implantado no Ministério da Justiça (MJ) do Brasil, para a gestão de documentos e processos eletrônicos e a fim de melhorar a eficiência administrativa no órgão. Diante disso, o objetivo da pesquisa é investigar a atitude de usuários em relação ao sistema SEI adotado no MJ, com base nas dimensões de adoção de tecnologia propostas por Moore e Benbasat (1991). Considerando ainda a aplicação de um instrumento adaptado desses autores, verifica-se, adicionalmente, a aplicabilidade das medidas adotadas no instrumento de coleta que foi construído à luz do instrumento original utilizado pelos autores, considerando-se os cinco atributos da inovação de Rogers (1983).

Nesse sentido, a pesquisa oferece contribuição teórica e empírica no que se refere ao estudo de adoção e difusão de tecnologias. A aplicação in loco do questionário adaptado do instrumento proposto por Moore e Benbasat (1991) amplia a discussão teórica do modelo já aplicado e com evidências de validade - sendo que o instrumento encontrou apoio também em outros estudos, como os de Agarwal e Prasad (1997, 1998), Karahanna, Straub e Chervany (1999) e Plouffe, Hulland e Vandenbosch (2001). Ademais, a perspectiva de usuários sobre o processo de adoção de um sistema no MJ gera insumos para que os gestores possam analisar cenários semelhantes ao deste estudo de caso e aperfeiçoar suas decisões relacionadas à adoção de sistemas gerenciais em organizações públicas. 


\section{Referencial teórico}

Esta seção apresenta breve panorama da discussão teórica sobre a tecnologia da informação nas organizações, a aceitação de tecnologias e os atributos da inovação e, por fim, destaca alguns estudos desenvolvidos a partir da abordagem de adoção de tecnologias de Moore e Benbasat (1991), autores centrais deste trabalho.

Segundo Bouwman et al. (2005), a tecnologia tem um papel fundamental no alcance dos objetivos de qualquer organização, pois ela ajuda a torná-los explícitos, a propagá-los e a medi-los. A tecnologia colabora com o aumento da eficiência e da eficácia organizacional ao cortar, por exemplo, custos de pessoal, encurtar o tempo de realização de um processo de trabalho e, ainda, criar novas formas de relação entre organização, fornecedores e clientes (BouWMAN et al., 2005).

Ribeiro Neto (1999) lembra que o impacto de novas tecnologias nas organizações pode ser percebido como mudanças em diferentes variáveis, tais como: conteúdo e natureza das tarefas, aptidão requerida, pressões e ritmo de trabalho, interação entre os funcionários, quantidade de funcionários e duração das jornadas de trabalho. Rodrigues (1988) destaca importantes efeitos da tecnologia da informação (TI) sobre as rotinas de trabalho e funcionários, como, por exemplo, a mudança das relações do funcionário com suas atividades e a possibilidade de provocar impacto sobre o nível de emprego, estresse e satisfação com o trabalho, além de exigir novas habilidades do funcionário. Já para Miranda et al. (2016), a adoção de sistemas nas organizações sem fins lucrativos pode resultar em melhorias relatadas como "otimização" de processos, referindo-se ao menor esforço causado pela redução de tarefas manuais e aumento de tarefas automatizadas que resultaram em velocidade no desempenho das tarefas. Participantes da pesquisa dessas autoras destacaram a melhoria dos processos por meio da agilidade. Ao buscar aperfeiçoamento e melhorias, as organizações vislumbram na reorganização de seus processos uma saída para acatar as pressões por corte de custos e incremento do uso de recursos internos.

No setor público, adquirir novas tecnologias e implementar novos sistemas tende a ser mais complicado do que em organizações privadas, como demonstrado por Colville e Carter (2013). Nos resultados de seu estudo, verificam-se os principais obstáculos mencionados pelos usuários: políticas e procedimentos muito rígidos, falta de incentivos e sistemas ineficientes de trabalho. Isso resulta em uma má performance da organização e na realização de muitas reformas organizacionais de curto prazo, que são alteradas antes mesmo de serem totalmente implementadas e estabelecidas, o que gera instabilidade à estratégia do órgão e muitos projetos acabam sendo prejudicados ou abandonados (COLVILLE; CARTER, 2013). 
A adoção de tecnologias no setor público pode trazer alguns benefícios importantes para o aumento da eficiência e melhoria da relação organizaçãoambiente, como aqueles levantados em estudo realizado por Deus e Farias (2015) acerca da adoção do sistema e-TCU pelo TCU: melhor controle do tempo, pela agilidade promovida pelo sistema; controle de custos; melhor controle da informação, do trabalho, da localização, do espaço, da imagem institucional e do controle ambiental. Deve-se ainda considerar o que os sujeitos, gestores do TCU, relataram sobre os atributos facilitadores de adoção da inovação naquele órgão, e que são propostos por Rogers (2003), a saber: vantagem relativa, compatibilidade, complexidade, testabilidade e observabilidade - fatores sempre relacionados positivamente com a tecnologia adotada, o que pode ter contribuído para o sucesso do processo de adoção e implantação do e-TCU.

A adoção de tecnologias já vem sendo estudada há algumas décadas, com o emprego de diferentes modelos teóricos que se propõem a explicar o processo de adoção no nível individual e organizacional (DOS SANTOS, 2007). Segundo Venkatesh, Morris e Davis (2003), estudos que envolvem a aceitação de novas tecnologias pelo usuário são frequentemente descritos como uma das áreas de pesquisa mais maduras na literatura de sistemas de informação, pois já há muito tempo pesquisas buscam responder como e por que indivíduos adotam novas tecnologias da informação. Assim, enseja-se a necessidade de trazer à discussão a questão atitudinal e comportamental dos indivíduos.

Segundo Eagly e Chaiken (1998), atitude é uma tendência psicológica que se expressa através da avaliação de uma situação particular de forma favorável ou desfavorável. Neste estudo, assume-se que identificar a atitude de usuários frente à adoção de um sistema é fundamental para estudar aspectos relativos à aceitação de tecnologias nas organizações.

A Teoria da Difusão da Inovação (TDI) tem Everett Rogers como referência, quando da publicação do livro Diffusion of Innovations em 1962. Segundo o autor, difusão é o processo pelo qual uma inovação é comunicada através de certos canais ao longo do tempo entre os membros de um sistema social. Constitui um tipo especial de comunicação, em que as mensagens estão relacionadas com novas ideias (Rogers, 1983). A nova ideia discutida neste trabalho é a adoção de TI em uma organização pública do setor executivo brasileiro. Este sistema de informação (SEI) estava em fase inicial de adoção quando da realização deste estudo em 2015.

A TDI de Rogers (1983) traz quatro aspectos que devem ser observados para entender as condições prévias da adoção da inovação, a saber: práticas anteriores, problemas/necessidades percebidas, grau de inovação e as normas do sistema social. Segundo o autor, o sistema social deve ser entendido como o contexto em 
que ocorre o processo de difusão. Após entender as condições prévias, o processo passa, então, por cinco estágios:

1. Conhecimento: ocorre quando o indivíduo ou unidade decisória é exposto a uma inovação e toma conhecimento de como ela funciona. A busca por informação acerca de uma inovação é feita essencialmente nesta fase, quando a organização e seus membros procuram saber de suas vantagens e desvantagens.

2. Persuasão: ocorre quando o indivíduo ou unidade decisória forma uma atitude favorável ou desfavorável em relação à inovação. Nesta etapa, os membros da organização já estão mais familiarizados com a inovação e passam a buscar novas informações de forma mais ativa e espontânea. Saber de atributos como vantagem relativa, compatibilidade e complexidade da tecnologia é extremamente importante neste estágio. É aqui que será feita uma projeção do futuro e um planejamento das ações vindouras.

3. Decisão: ocorre quando o indivíduo ou unidade decisória toma atitudes que irão culminar na decisão de adotar ou não a inovação. É nesta fase que geralmente ocorrem os testes e verificações da nova tecnologia, que ajudam a reduzir o grau de incerteza do usuário. Nos casos em que não é possível realizar testes prévios, a adoção tende a ser lenta, inclusive com mais chances de rejeição. Rogers (1983) argumenta que quanto maior a vantagem relativa da inovação, mais cedo ela será adotada.

4. Implementação: ocorre quando o indivíduo ou unidade decisória coloca em uso a inovação. Até este estágio, o processo de adoção de tecnologia é estritamente mental, mas aqui se faz necessária uma mudança comportamental, pois é quando a nova ideia é colocada em prática.

5. Confirmação: ocorre quando o indivíduo ou unidade decisória reforça a adoção da tecnologia. Mas essa decisão pode mudar caso surjam conflitos questionando essa tecnologia. O estágio de confirmação continua mesmo depois da decisão de adotar ou não, por um período indeterminado de tempo.

Rogers (1983) sugere cinco características pelas quais uma inovação pode ser descrita ou avaliada, a partir da verificação das atitudes dos indivíduos em relação a atributos, a saber:

1. Vantagem relativa: que é quanto uma inovação é percebida como sendo melhor do que a ideia que ela substitui. A vantagem relativa de uma inovação, como percebida pelos membros de um sistema social, é positivamente relacionada com a sua taxa de adoção. 
2. Compatibilidade: que é quanto uma inovação é percebida como consistente com os valores existentes, experiências passadas e as necessidades de potenciais usuários. A compatibilidade de uma inovação, assim como a vantagem relativa, é positivamente relacionada com a sua taxa de adoção.

3. Complexidade: que é quanto uma inovação é percebida como difícil de entender e de usar. A complexidade de uma inovação, como percebida pelos membros de um sistema social, é negativamente relacionada com a sua taxa de adoção.

4. Testabilidade: que é quanto uma inovação pode ser testada. Quanto maior o nível de testabilidade, maior será a taxa de adoção da inovação.

5. Observabilidade: que é quanto os resultados da inovação são visíveis para os indivíduos. A observabilidade de uma inovação é positivamente relacionada à sua taxa de adoção.

A taxa de adoção é a velocidade relativa com que uma inovação é adotada por membros de um sistema social (RogERS, 1983, p. 232). Geralmente ela é medida pelo número de indivíduos que adotam uma nova ideia em um determinado período de tempo. O processo de difusão de Rogers (1983) é permeado por fatores organizacionais e individuais que vão dificultar ou contribuir para o sucesso e fluidez do processo e sua posterior consolidação.

Moore e Benbasat (1991) testaram empiricamente os cinco atributos da inovação em uma pesquisa cujo objetivo era desenvolver uma escala para medir as várias atitudes frente ao uso de computadores. A intenção dos autores era criar uma escala que pudesse ser aplicada em uma grande variedade de inovações, especialmente quando relacionadas à tecnologia da informação. Como resultado, o instrumento da referida pesquisa pôde ser usado, com algumas adaptações, em vários estudos sobre difusão de inovações.

Para este estudo, foi seguido o modelo de Moore e Benbasat (1991), que aborda os construtos de vantagem relativa; compatibilidade; facilidade de uso; demonstrabilidade dos resultados; e testabilidade. Dessa forma, como já explicitado na introdução do artigo, este estudo propõe, como um objetivo secundário, aplicar questionário adaptado daquele proposto por Moore e Benbasat (1991), ou seja, verificar a aplicabilidade das medidas adotadas no instrumento de coleta que foi construído à luz do instrumento original utilizado por esses autores. Assim, foi possível verificar quão confiáveis e consistentes se apresentam as medidas utilizadas à aplicação no lócus escolhido para esta pesquisa.

Como o trabalho enfocou a contribuição inicial de Moore e Benbasat (1991), visando trabalhar com o instrumento original adaptado dos autores, os parágrafos 
seguintes elucidam um pouco mais o nível de utilização e pertinência do uso do instrumento desses precursores em pesquisas que tratam da adoção de tecnologias. Sendo assim, alguns estudos são destacados a seguir, com a ressalva de que a abordagem é quantitativa ou qualitativa, embora utilizem as dimensões de análise de Rogers (1983) e de Moore e Benbasat (1991) no processo de análise das evidências empíricas.

Em sua pesquisa, Leal (2012) teve como objetivo identificar fatores que determinam o uso de inovação tecnológica na educação a distância, na percepção de docentes atuantes em cursos na área de negócios. Com o instrumento desenvolvido a partir do modelo de Moore e Benbasat (1991), porém sob influência de vários outros teóricos, o estudo testou a validade e confiabilidade dos seguintes construtos: vantagem relativa, compatibilidade, facilidade de uso, experimentação, visibilidade, imagem, demonstração de resultado e uso voluntário, sendo o objetivo principal verificar o quanto essas variáveis influenciavam o uso da inovação tecnológica estudada. Como resultado, constatou-se que os atributos que afetam diretamente o uso dos ambientes virtuais de aprendizagem são a compatibilidade, a vantagem relativa e a demonstração de resultado (LEAL, 2012).

Pinsky (2009) realizou um survey com 271 professores de duas faculdades, optando por se basear no instrumento de Moore e Benbasat (1991), alegado como o mais adequado à sua pesquisa, que teve como objetivo identificar diferenças de percepções sobre a adoção do livro didático eletrônico entre professores universitários e profissionais de editoras (PINSKY, 2009). No referido estudo, concluiu-se que, no geral, os professores não apresentam diferenças significativas de percepção quanto ao livro eletrônico, quando comparados com grupos de docentes da USP e da Mackenzie. Ainda assim, para cinco das variáveis estudadas, houve diferenças entre grupos. Considerando-se 29 itens do instrumento, em 14 deles se verificaram diferenças entre grupos, quando utilizadas as variáveis propostas no estudo seminal de Moore e Benbasat (1991), a saber: vantagem relativa, compatibilidade, experimentação, facilidade de uso, imagem, uso voluntário, visibilidade e demonstração de resultados.

O trabalho qualitativo desenvolvido por Perez et al. (2010) teve como objetivo responder à pergunta de pesquisa: "Quais fatores percebidos pelos usuários contribuem para a adoção de sistemas de informações na área de saúde?", definindose como tecnologia estudada o prontuário eletrônico de um pronto-socorro infantil e um sistema de apoio ao ensino pela web em uma faculdade de medicina. Os pesquisadores verificaram que os respondentes mostraram atitude positiva com relação aos aspectos relacionados ao sistema social e às variáveis identificadas por Rogers (2003). Em um esforço de análise da adequação de um modelo de escala 
multi-itens, visando avaliar fatores que determinavam o uso de inovação tecnológica na educação a distância na percepção de docentes de Administração e de Ciências Contábeis, Leal e Albertin (2015) realizaram uma análise fatorial confirmatória, utilizando-se dos construtos propostos por Rogers (1983), a saber: vantagem relativa, compatibilidade, complexidade, experimentação e observabilidade. Quanto ao atributo complexidade, houve sua substituição por facilidade de uso, e de observabilidade por visibilidade, evidenciando-se, já, a influência de Moore e Benbasat (1991). Posteriormente, em um grupo 2 (modelo 2), os autores incluíram mais cinco construtos, sendo três de Moore e Benbasat (1991): imagem, uso voluntário (voluntariedade) e demonstração de resultado. Por fim, os outros dois construtos incluídos foram: o domínio tecnológico e uso de inovação tecnológica. Segundo os autores, o trabalho alcançou a validade convergente e discriminante, e todos os construtos investigados se mostraram consistentes e confiáveis.

Finalizando a discussão teórica, esclarece-se que o que os autores aqui citados denominam como experimentação, nesta pesquisa ora apresentada preferiu-se aproximar do termo trialability por meio de neologismo, adotandose a palavra testabilidade.

\section{Método}

A pesquisa teve uma abordagem quantitativa com perspectiva transversal e natureza descritiva, em que houve a preocupação de verificar a atitude de servidores do Ministério da Justiça (MJ) quanto à adoção de tecnologias, sendo que a tecnologia estudada foi o Sistema Eletrônico de Informações (SEI). Todos os servidores já possuíam login e senha para acesso ao SEI, plataforma web onde produzem documentos eletrônicos, assinam digitalmente, tramitam entre diferentes unidades e arquivam eletronicamente, quando da finalização do processo. Ou seja, mais do que um sistema de tramitação de documentos, o SEI é uma ferramenta de trabalho, pois todos os documentos oficiais são produzidos no próprio sistema. Com a adoção do SEI no MJ, todos os processos criados a partir de 2015 passaram a ser exclusivamente eletrônicos, o que reduziu enormemente a utilização de papel na organização e representou uma mudança de paradigma no órgão.

Todos os 600 usuários do SEI no MJ foram convidados a participar da pesquisa. A taxa de resposta foi de $34,7 \%$, atingindo-se 208 respondentes ao instrumento de pesquisa. Destes, 52,4\% eram servidores no MJ há mais de três anos e $60,1 \%$ usavam o sistema há pelo menos cinco meses. Cabe salientar que à época da coleta dos dados o SEI estava em execução no MJ há seis meses. Os usuários tinham idade entre 17 e 64 anos, com média de 36,16 anos ( $D P=10,13), 51,0 \%$ eram do sexo feminino e a maioria possuía nível superior completo $(79,2 \%)$. 
Para a aplicação da pesquisa foi utilizado um instrumento com a escala de Moore e Benbasat (1991), traduzida para o português, com 26 itens apresentados em estrutura tipo-Likert de concordância, com sete pontos. A estrutura original da escala aplicada em inglês no Canadá apresentou retenção de cinco fatores com alfas de Cronbach de 0,92 para vantagem relativa (VR), 0,83 para compatibilidade (CP), 0,80 para facilidade de uso (FU), 0,77 para demonstrabilidade dos resultados (DR) e 0,71 para testabilidade (TE). Adicionalmente, houve cinco questões sócio demográficas que compreenderam o levantamento de: tempo de uso do SEI, tempo de trabalho no MJ, idade, sexo e escolaridade dos respondentes.

Para a coleta dos dados, foi formalizado um pedido dos pesquisadores junto ao Ministério da Justiça, no qual se retratava o interesse de estudar o SEI naquela organização. Após a autorização do MJ, o questionário foi aplicado, em parceria com o Grupo de Trabalho do SEI instituído pelo MJ para atuar na implantação do sistema. Os dados foram coletados por intermédio de um questionário online, elaborado e disponibilizado na plataforma Google Forms. Esse questionário foi enviado por e-mail aos usuários do SEI com um link convidando-os a participar da pesquisa. A coleta ocorreu ao longo do mês de maio de 2015.

\section{Resultados}

Para investigar a atitude de usuários em relação ao sistema SEI adotado no MJ, os resultados foram subdivididos em duas etapas. Na primeira etapa, foi executada uma análise fatorial exploratória (AFE) da escala adaptada de Moore e Benbasat (1991), com a finalidade de identificar evidências de validade da escala para o Brasil. Na segunda etapa, considerando a não normalidade dos dados, foram conduzidos testes não paramétricos de comparação entre grupos, com a finalidade de avaliar a adoção de tecnologia em relação às características sociodemográficas dos usuários do sistema.

Como parte da AFE, a extração inicial dos dados pelo método de componentes principais identificou um KMO de 0,94. O método do gráfico de sedimentação (teste scree de Cattell), pelo critério de análise dos autovalores, sugeriu a retenção de quatro a cinco fatores. Foram testadas diversas extrações pelo método dos eixos principais (PAF) com rotação oblíqua direta e oblíqua promax, bem como com rotação ortogonal varimax. Esta última seguindo a análise conduzida no estudo de Moore e Benbasat (1991). A melhor solução foi com rotação oblíqua promax, para quatro fatores, explicando ao todo $71,46 \%$ da variância. Quatro itens não compuseram nenhum fator por apresentarem carga fatorial abaixo de 0,45, seguindo orientações sugeridas por Churchill (1979) e Lee e Hooley (2005), ou por apresentarem comunalidade abaixo de 0,50 (HAIR et al., 2005). Assim, a escala, 
para a amostra analisada, totalizou 22 itens. Sendo que os itens de vantagem relativa (VR) e compatibilidade (CP) se misturaram fortemente e compuseram um único fator. Os demais fatores obedeceram a estrutura teórica sugerida por Moore e Benbasat (1991).

Os quatro fatores foram denominados como: (1) VRCP $(\alpha=0,97)$, com 12 itens relacionados aos aspectos de vantagem relativa e compatibilidade; (2) FU ( $\alpha=$ $0,78)$, com quatro itens relacionados aos aspectos de facilidade de uso; (3) TE $(\alpha=0,85)$, com quatro itens relacionados aos aspectos de testabilidade; (4) DR $(\alpha=0,87)$, com dois itens relacionados aos aspectos de demonstrabilidade dos resultados. Assim, a estrutura fatorial final da escala de adoção de tecnologia em relação ao SEI/MJ está apresentada no Quadro 1, com os itens e respectivas cargas fatoriais ordenadas por fator.

Quadro 1 - Escala de adoção de tecnologia em relação ao SEI/MJ

\begin{tabular}{|c|c|c|}
\hline FATOR & ITEM & $\begin{array}{l}\text { Carga } \\
\text { Fatorial }\end{array}$ \\
\hline \multirow{12}{*}{$\begin{array}{l}\text { Vantagem relativa e } \\
\text { compatibilidade } \\
\text { (VRCP) }\end{array}$} & $\begin{array}{l}\text { VR2. O uso do SEI melhora a qualidade do } \\
\text { meu trabalho }\end{array}$ & 0,95 \\
\hline & $\begin{array}{l}\text { VR8. O uso do SEI aumenta a minha } \\
\text { produtividade }\end{array}$ & 0,91 \\
\hline & $\begin{array}{l}\text { VR6. O uso do SEI melhora minha eficiência } \\
\text { no trabalho }\end{array}$ & 0,90 \\
\hline & $\begin{array}{l}\text { CP10. O uso do SEl é totalmente compatível } \\
\text { com a situação na qual me encontro }\end{array}$ & 0,90 \\
\hline & $\begin{array}{l}\text { VR1. O uso do SEI permite realizar minhas } \\
\text { tarefas mais rapidamente }\end{array}$ & 0,88 \\
\hline & $\begin{array}{l}\text { VR4. O uso do SEI melhora meu } \\
\text { desempenho no trabalho }\end{array}$ & 0,87 \\
\hline & $\begin{array}{l}\text { CP11. Acho que o uso do SEl se encaixa bem } \\
\text { com o modo como gosto de trabalhar }\end{array}$ & 0,84 \\
\hline & $\begin{array}{l}\text { VR5. No geral, acho que o uso do SEl é } \\
\text { vantajoso para o meu trabalho }\end{array}$ & 0,83 \\
\hline & $\begin{array}{l}\text { CP9. O uso do SEl é compatível com todos os } \\
\text { aspectos do meu trabalho }\end{array}$ & 0,83 \\
\hline & $\begin{array}{l}\text { CP12. O uso do SEI se encaixa ao meu estilo } \\
\text { de trabalho }\end{array}$ & 0,81 \\
\hline & $\begin{array}{l}\text { VR3. O uso do SEI permite que realize meu } \\
\text { trabalho mais facilmente }\end{array}$ & 0,78 \\
\hline & $\begin{array}{l}\text { VR7. O uso do SEI me dá maior controle } \\
\text { sobre o meu trabalho }\end{array}$ & 0,76 \\
\hline
\end{tabular}




\begin{tabular}{|c|c|c|}
\hline FATOR & ITEM & $\begin{array}{l}\text { Carga } \\
\text { Fatorial }\end{array}$ \\
\hline \multirow{4}{*}{ Facilidade de uso } & $\begin{array}{l}\text { FU14. Preciso de muito esforço mental } \\
\text { quando estou usando o SEI }\end{array}$ & 0,85 \\
\hline & FU13. Acho o SEI difícil de usar & 0,66 \\
\hline & $\begin{array}{l}\text { FU15. O uso do SEI é frequentemente } \\
\text { frustrante }\end{array}$ & 0,60 \\
\hline & FU17. No geral, acho que o SEl é fácil de usar & 0,50 \\
\hline \multirow{4}{*}{ Testabilidade } & $\begin{array}{l}\text { TE25. O SEI esteve disponível para mim para } \\
\text { assim poder testar adequadamente suas } \\
\text { funcionalidades }\end{array}$ & 0,88 \\
\hline & $\begin{array}{l}\text { TE26. Antes de decidir quando utilizar } \\
\text { algumas das funcionalidades do SEI, pude } \\
\text { testá-las apropriadamente }\end{array}$ & 0,80 \\
\hline & $\begin{array}{l}\text { TE24. Eu sei o que devo fazer para poder } \\
\text { testar as várias utilidades do SEI }\end{array}$ & 0,77 \\
\hline & $\begin{array}{l}\text { TE23. Eu tive a oportunidade de testar várias } \\
\text { funcionalidades do SEI }\end{array}$ & 0,61 \\
\hline $\begin{array}{l}\text { Demonstrabilidade } \\
\text { de resultados }\end{array}$ & $\begin{array}{l}\text { DR20. Acredito que poderia compartilhar } \\
\text { com os outros as consequências de usar o } \\
\text { SEI }\end{array}$ & 0,79 \\
\hline (DR) & $\begin{array}{l}\text { DR19. Eu não teria dificuldade em falar para } \\
\text { os outros sobre os resultados causados pelo } \\
\text { uso do SEI }\end{array}$ & 0,67 \\
\hline
\end{tabular}

Comparando a pesquisa original desenvolvida por Moore e Benbasat (1991), com cada alfa $(\alpha)$ obtido nesta pesquisa, é possível concluir que as medidas adotadas no instrumento adaptado à aplicação no Brasil para esta pesquisa possuem significativa convergência com o trabalho original, pois os valores de alfa são bastante aproximados ou superiores àqueles do instrumento de Moore e Benbasat (1991). Isso pode ser constatado na Tabela 1. 
Tabela 1 - Alfa de Cronbach das medidas relativas aos construtos

\begin{tabular}{lccc} 
& $\begin{array}{c}\text { Alfa de Cronbach } \\
\text { desta pesquisa } \\
\text { (2015) }\end{array}$ & $\begin{array}{c}\text { Alfa Cronbach } \\
\text { da pesquisa } \\
\text { original (MooRE; } \\
\text { BENBASAT, 1991) }\end{array}$ & $\begin{array}{c}\text { No de itens por } \\
\text { fator }\end{array}$ \\
\hline VR = Vantagem relativa & - & 0,92 & - \\
\hline CP = Compatibilidade & - & 0,83 & 4 \\
\hline FU = Facilidade de uso & 0,83 & 0,80 & 2 \\
\hline $\begin{array}{l}\text { DR = Demonstrabilidade } \\
\text { dos resultados }\end{array}$ & 0,87 & 0,77 & 4 \\
\hline $\begin{array}{l}\text { TE }=\text { Testabilidade } \\
\text { VRCP = Junção ocorrida } \\
\text { neste estudo entre os } \\
\text { dois fatores VR+CP }\end{array}$ & 0,85 & 0,71 & 12 \\
\hline \begin{tabular}{l} 
TOTAL \\
\hline
\end{tabular} & 0,97 & - & 22 \\
\hline
\end{tabular}

Fonte: elaboração própria.

Com relação à avaliação que os usuários do $\mathrm{MJ}$ fazem do SEI, foi identificado que, de maneira geral, eles concordam menos com as assertivas ligadas ao fator testabilidade e concordam mais com os fatores relacionados à facilidade de uso, como mostra a Tabela 2.

Tabela 2 - Análise descritiva dos fatores de adoção de tecnologia em relação ao $\mathrm{SEI} / \mathrm{MJ}$

\begin{tabular}{lccccc}
\hline & Média & Mediana & DP & Min & Max \\
\hline VRCP & 5,37 & 5,75 & 1,52 & 1,00 & 7,00 \\
\hline FU & 5,53 & 5,75 & 1,29 & 1,00 & 7,00 \\
\hline TE & 4,49 & 4,50 & 1,51 & 1,00 & 7,00 \\
\hline DR & 5,55 & 6,00 & 1,41 & 1,00 & 7,00 \\
\hline
\end{tabular}

Fonte: elaboração própria. 
Em seguida, considerando variáveis sociodemográficas levantadas no estudo - sexo do respondente, escolaridade, tempo de uso do SEI, idade do usuário e tempo de trabalho no $\mathrm{MJ}$-, foi executada análise de comparação entre grupos. Assim, aplicando o teste não paramétrico de Mann-Whitney (U), não se verificaram diferenças quanto à avaliação do SEI para a variável sexo. O mesmo aconteceu quando considerada a variável escolaridade, usando o teste não paramétrico de Kruskal-Wallis.

Para avaliar a adoção de tecnologia pelos usuários em relação ao tempo de uso do SEI, foram criados dois grupos: aqueles que utilizavam o SEI há pelo menos 5 meses ( $\mathrm{N}=125)$ e, portanto, com contato mais amplo com o sistema; e aqueles com até 4 meses de uso do sistema ( $N=83$ ). É importante lembrar que esse corte temporal se deveu ao fato de o SEI ter sido implementado há apenas seis meses, à época da coleta dos dados. Nesse sentido, pelo teste de Mann-Whitney $U$, os resultados foram significativos apenas para as variáveis $\operatorname{VRCP}(U=6.037,00, p<0,05)$ e TE $(U=6.054,50, p<0,05)$. A variação da mediana dos fatores VRCP e TE em relação ao tempo de uso do SEI pode ser visualizada na Figura 1.

Figura 1 - Variação da mediana dos fatores VRCP e TE em relação ao tempo de uso do SEI

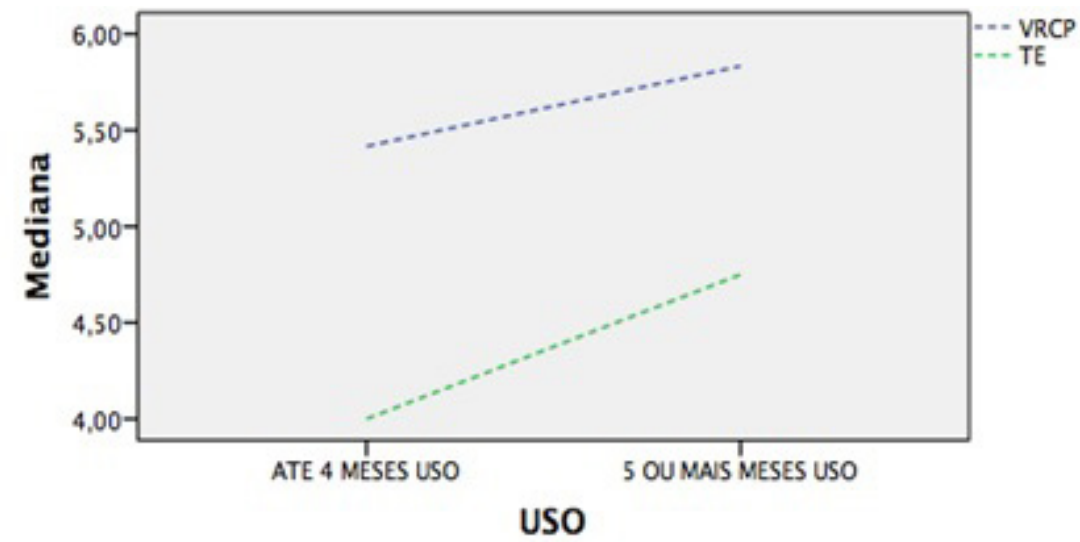

Fonte: elaboração própria.

Com relação à idade do usuário, foram criadas três faixas etárias, balanceadas pela distribuição da amostra. Assim, foi obtida uma faixa de usuários com idade até 30 anos $(\mathrm{N}=70)$, entre 31 e 40 anos $(\mathrm{N}=81)$, e mais de 40 anos $(\mathrm{N}=57)$. Para essas faixas, pelo teste de Kruskal-Wallis, houve diferença significativa para todos os fatores, exceto VRCP. Os resultados significativos na análise podem ser observados 
na Tabela 3.

Tabela 3 - Comparação dos fatores de adoção da tecnologia em relação à faixa etária dos usuários

\begin{tabular}{|c|c|c|c|c|c|c|}
\hline \multirow[b]{2}{*}{ Fator } & \multirow{2}{*}{$\begin{array}{c}\text { Kruskal- } \\
\text { Wallis }\end{array}$} & \multirow[b]{2}{*}{$p$} & \multirow[b]{2}{*}{ Faixa etária } & \multirow[b]{2}{*}{ Mediana } & \multicolumn{2}{|c|}{ Análise post hoc* } \\
\hline & & & & & $\begin{array}{c}31 \text { a } 40 \\
\text { anos }\end{array}$ & $\begin{array}{c}\text { Mais } 40 \\
\text { anos }\end{array}$ \\
\hline \multirow[t]{3}{*}{ FU } & 9,49 & 0,009 & $\begin{array}{l}\text { Até } 30 \\
\text { anos }\end{array}$ & 5,62 & 0,023 & - \\
\hline & & & $\begin{array}{c}31 \text { a } 40 \\
\text { anos }\end{array}$ & 6,25 & - & 0,031 \\
\hline & & & $\begin{array}{c}\text { Mais } 40 \\
\text { anos }\end{array}$ & 5,50 & - & - \\
\hline \multirow[t]{3}{*}{ TE } & 8,48 & 0,014 & $\begin{array}{l}\text { Até } 30 \\
\text { anos }\end{array}$ & 4,50 & - & - \\
\hline & & & $\begin{array}{c}31 \text { a } 40 \\
\text { anos }\end{array}$ & 5,00 & - & - \\
\hline & & & $\begin{array}{c}\text { Mais } 40 \\
\text { anos }\end{array}$ & 4,00 & - & 0,011 \\
\hline \multirow[t]{3}{*}{$\mathrm{DR}$} & 8,60 & 0,014 & $\begin{array}{l}\text { Até } 30 \\
\text { anos }\end{array}$ & 5,50 & 0,029 & - \\
\hline & & & $\begin{array}{c}31 \text { a } 40 \\
\text { anos }\end{array}$ & 6,00 & - & - \\
\hline & & & $\begin{array}{c}\text { Mais } 40 \\
\text { anos }\end{array}$ & 6,00 & - & - \\
\hline
\end{tabular}

Fonte: elaboração própria.

Nota. (*) Apenas valores significativos, com a aplicação da correção de Bonferroni.

Por fim, analisaram-se os fatores de adoção da tecnologia em relação ao tempo de trabalho do usuário no MJ. Obedecendo ao mesmo critério de criação das faixas de tempo balanceadas pela distribuição da amostra, tem-se: usuários com até um ano de trabalho no $\mathrm{MJ}(\mathrm{N}=45)$, usuários com 1 a 3 anos de trabalho $(\mathrm{N}=54)$, usuários com 3 a 5 anos $(\mathrm{N}=57)$ e usuários com mais de 5 anos $(\mathrm{N}=52)$. Entre as faixas de tempo de trabalho, apenas foram identificadas diferenças significativas para os fatores $\operatorname{VRCP}(H=7,83, p<0,05)$ e $F U(H=11,36, p<0,05)$. Na análise post hoc, apenas os grupos de 1 a 3 anos versus o grupo de 3 a 5 anos apresentaram diferença significativa. 


\section{Discussão}

Como se pôde notar na Tabela 1 , somente neste estudo os itens de vantagem relativa (VR) e compatibilidade (CP) se mesclaram intensamente, compondo um só fator (VRCP). Os demais fatores obedeceram à estrutura teórica sugerida por Moore e Benbasat (1991). Isso pode sinalizar a necessidade de duas reflexões a serem feitas em estudos posteriores: 1) pode ter havido problemas semânticos na construção das assertivas relativas aos itens componentes das duas dimensões VR e CP, o que as fez, neste trabalho, agruparem-se; 2 ) a variável compatibilidade (CP) integrou-se à vantagem relativa (VR), pois, talvez, tenha se tornado uma subcategoria, visto que, ao analisar vantagens da adoção de uma tecnologia, é compreensível que o adotante possa analisar, entre os atributos, a compatibilidade. Resta explorar essa reflexão a posteriori, buscando reavaliar quão abrangente seria a variável VR diante das outras, com vistas à identificação de eventual subordinação das demais. No estudo de Hung et al. (2010), observou-se que, ao estudarem as características de um customer relationship management system (CRMS) em hospitais e definir variáveis influenciadoras de sua adoção, os autores consideraram apenas duas variáveis diante do conjunto proposto por Rogers (1983), a saber: vantagem relativa e complexidade. A variável compatibilidade não aparece no modelo dos autores como uma variável adicional. Simplesmente não foi considerada no modelo preditivo. Abre-se aqui uma oportunidade ao aprofundamento de reflexões sobre o quanto a variável VR pode ser uma espécie de variável abarcadora dos demais atributos ou características de inovações propostos em 1963 por Rogers e adotados, posteriormente, por Moore e Benbasat (1991) e por vários outros pesquisadores até a atualidade.

A respeito dos resultados relativos à atitude de usuários do SEI que participaram da pesquisa, concernente aos atributos da tecnologia analisada, o fato de a Tabela 2 demonstrar que os sujeitos concordam menos com as assertivas ligadas ao fator testabilidade e concordam mais com os fatores relacionados à facilidade de uso pode ser explicado por uma espécie de ação recorrente de organizações quando decidem implantar novos sistemas. A decisão, muitas vezes, é mandatória e com pouco prazo para a efetiva implantação. O processo de adoção não é um processo sequencial, linear, em que se dedique um tempo maior para o treinamento e/ ou testes exaustivos antes da adoção da tecnologia. Tornatzky e Fleischer (1990) destacam a característica de iteratividade e não linearidade do processo de adoção de tecnologias. Assim, embora fácil de utilizar, é compreensível que as pessoas destaquem o pouco tempo dedicado ao preparo (testes etc.) para o uso do sistema.

Quanto à média das variáveis, facilidade de uso (FU) apresentou a maior média. Portanto, as assertivas referentes a essa variável captaram maior grau de 
concordância entre os respondentes, o que reflete uma atitude positiva de usuários do SEI frente à vantagem comparativa do sistema. Com exceção de testabilidade $(\mathrm{TE})$, todas as variáveis apresentaram média acima de 5 . Ainda quanto à variável testabilidade (TE), cuja média foi a menor dentre todas, quando se analisou o Alfa de Cronbach, conforme se visualiza na Tabela 1, houve grande discrepância do alfa extraído da análise do instrumento original de Moore e Benbasat (1991). Portanto, pode ser pouco provável que o problema tenha relação com problemas semânticos dos itens, visto que o alfa foi superior ao da pesquisa original.

Adicionalmente, é importante destacar que o trabalho original de Moore e Benbasat (1991) adotou uma solução fatorial com rotação ortogonal, que é indicada para fatores com baixa ou nenhuma correlação entre si, como esclarece Pasquali (2012). No entanto, os fatores que compõem a estrutura da escala de adoção de tecnologia são extremamente correlacionados e exigem o uso de rotação oblíqua, que foi exatamente a melhor solução encontrada no presente estudo, com alfas também melhores.

Outras análises foram conduzidas com o intuito de melhor conhecer os sujeitos do estudo e eventuais diferenças entre grupos. Para tanto, procedeu-se, conforme apresentado na seção de resultados (Figura 1 e Tabela 3), a alguns testes de comparações múltiplas que serão comentados a seguir.

Agarwal e Prasad (1999) sugerem que as diferenças individuais tais como gênero, idade, nível de escolaridade e experiência podem ser utilizadas para construir um perfil dos indivíduos mais receptivos às novas tecnologias. Tais autores ainda indicam que a aceitação pode ser facilitada pela utilização de outras intervenções que afetam diretamente a atitude dos sujeitos, como a formação e o desenvolvimento de uma cultura de aprendizagem na organização. Também Venkatesh, Thong e Xu (2012) destacam as variáveis gênero, idade e experiência com tecnologias - verificada a partir da frequência de uso - como preponderantes na moderação das relações entre intenção de usar, comportamento de uso e suas variáveis influenciadoras diretas, a exemplo da expectativa de performance, expectativa de esforço, entre outras. Assim, não se devem desprezar variáveis demográficas em estudos sobre adoção de tecnologias, especificamente quando se estuda a atitude dos sujeitos envolvidos.

Com relação à experiência, os resultados alcançados com o teste de MannWhitney $U$ e demonstrados na seção de resultados, bem como a variação da mediana dos fatores VRCP e TE em relação ao Tempo de Uso do SEI, visualizada na Figura 1, revelaram que há diferenças significativas apenas em relação às variáveis VRCP (vantagem relativa + compatibilidade) e TE (testabilidade), quando considerados usuários há pelo menos cinco meses e usuários com até quatro meses de uso do SEI. 
O tempo de uso de uma ferramenta tecnológica é discutido por Venkatesh, Thong e Xu (2012) como uma variável denominada experiência. Esta pode moderar a intenção e o comportamento humano no uso da tecnologia. Rogers (1983) afirma que um fator importante que impacta a taxa de adoção de qualquer inovação é a sua compatibilidade com os valores, crenças e experiências presentes no sistema social. Venkatesh e Bala (2008) acrescentam que, com o aumento da experiência, o efeito da variável facilidade de uso sobre a utilidade percebida tende a ser maior, ou seja, com o uso frequente de uma inovação por um indivíduo, é provável que ele gradativamente ache seu uso mais fácil e útil. Dessa maneira, nesta pesquisa, é possível concluir que os usuários que utilizam o sistema SEI há mais tempo diferem dos usuários mais recentes em termos de percepção de sua vantagem relativa (VR), compatibilidade (CP) e testabilidade (TE).

Venkatesh, Thong e Xu (2012) também discutem a importância de comparar fatores de adoção de tecnologia em relação à faixa etária dos usuários. A Tabela 3 que se encontra na seção anterior demonstrou que diferentes grupos por faixa etária manifestam diferenças de percepção em relação a três variáveis estudadas (FU, $T E, D R$ ). Pessoas mais jovens tendem a valorizar mais os resultados demonstráveis com o uso da ferramenta adotada (DR), enquanto pessoas mais velhas valorizam aspectos da facilidade de uso (FU) e as possibilidades de teste da tecnologia (TE). Grande quantidade de estudos já comprovou que a idade é fator que não pode ser desprezado em estudos sobre a adoção de tecnologias (VENKATESH et al., 2003; EMANI et al., 2012; LU; DenG; WANG, 2010; WATTAL; RACHERLA; MANDVIWALLA, 2010; VENKATESH; SYKES; VENKATRAMAN, 2014). Assim, os resultados deste estudo reforçam a importância de manter esta variável em pesquisas futuras.

\section{Considerações finais}

O objetivo central deste trabalho foi investigar a atitude de usuários em relação ao sistema SEI adotado no Ministério da Justiça, com base nas dimensões de adoção de tecnologia propostas por Moore e Benbasat (1991). Adicionalmente, considerando o emprego de um instrumento adaptado desses autores, buscou-se verificar a aplicabilidade das medidas adotadas no instrumento de coleta que foi construído à luz do instrumento original utilizado pelos autores, considerando-se também os cinco atributos da inovação de Rogers (1983).

Confirmou-se a confiabilidade das medidas adotadas na elaboração dos construtos utilizados no instrumento de pesquisa - vantagem relativa, compatibilidade, facilidade de uso, demonstrabilidade dos resultados e testabilidade - uma vez que todos apresentaram um Alfa de Cronbach superior a 0,7, evidenciando a adequação das medidas do instrumento proposto por Moore e Benbasat (1991) 
em estudos sobre adoção e difusão de inovações ligadas às tecnologias da informação. Entretanto, importa destacar que os itens relativos às dimensões vantagem relativa (VR) e compatibilidade (CP) juntaram-se, compondo um só fator (VRCP), recomendando-se na discussão que se aprofundem as investigações sobre a ocorrência de tal convergência.

No geral, a atitude dos sujeitos da pesquisa é favorável à adoção do SEI. De maneira mais específica, com a comparação entre grupos, foi possível concluir que usuários que utilizam o sistema SEI há mais tempo diferem de usuários iniciantes, quando avaliadas a vantagem relativa (VR), a compatibilidade (CP) e a testabilidade (TE) da tecnologia adotada.

Também foi possível confirmar o que a vasta literatura sobre a intenção e o comportamento de uso de tecnologias defende sobre a questão da idade. Pessoas mais velhas valorizam a facilidade de uso e a oportunidade de testar uma tecnologia, enquanto sujeitos mais jovens estão focalizados na demonstrabilidade de resultados da solução tecnológica adotada.

A expectativa, antes de iniciar o estudo, foi de que o sistema SEI fosse bem aceito pelos usuários e isso, de fato, confirmou-se nos resultados, pois o sistema foi bem recepcionado pelos usuários - mesmo entre os mais velhos, grupo em que normalmente há uma maior barreira à aceitação de inovações.

Fato relevante do estudo foi constatar que a transição do processo físico para o processo eletrônico representou uma mudança de paradigma na organização estudada e, surpreendentemente, tal processo não foi traumático. Observou-se que os usuários rapidamente se "desapegaram" dos documentos físicos, aceitando a nova forma de trabalho de maneira natural e acreditando que o SEI seria um aliado na desburocratização da máquina pública.

Para estudos futuros, recomenda-se um aprofundamento com vista a esclarecer por que duas variáveis convergiram a uma só neste estudo. Recomenda-se, ainda, a ampliação da aplicação do instrumento de pesquisa a outros órgãos públicos que estão adotando o SEI, visto que, embora na literatura o assunto esteja amadurecido quando se discute o objeto em organizações privadas, no Brasil, estudos dessa natureza no setor público e no governo ainda são escassos.

Quando considerados os âmbitos federal, estadual, municipal e estatal, tem-se 363 instituições públicas que resolveram adotar o SEI, das quais, até 2018, apenas 113 adotaram e já utilizam a tramitação eletrônica de documentos/processos (Ministério do Planejamento, Desenvolvimento e Gestão, 2018). Sendo assim, aproximadamente $70 \%$ ainda estão em processo de implantação do SEl, sendo que o prazo estabelecido no Decreto no 8.539/2015 para os órgãos da administração pública federal (APF) foi de dois anos para a adesão dos órgãos (pelo menos APF) à 
ferramenta tecnológica SEI ou qualquer outra que substituísse em definitivo o papel por sistemas informatizados utilizados no trato com os processos administrativos nos órgãos da APF. Sendo assim, a adoção do SEl continua sendo um tema que necessita de mais estudos, inclusive para que sejam levantados os desafios e barreiras à sua implementação no setor público.

Nesta pesquisa, foram ouvidos os usuários do SEI no MJ. Porém, dando continuidade ao projeto, também se levantou a percepção dos gestores envolvidos com a adoção e implementação do SEI naquele Ministério, em trabalho que se encontra em elaboração para publicação. Fatores relacionados com tecnologia, custos, infraestrutura e legitimação (apoio da alta gestão) também merecem um olhar de pesquisadores do tema, no âmbito da implantação do SEI na APF brasileira, uma vez que o assunto é complexo, multifacetado e ainda se pode considerar incipiente a iniciativa de estudos de adoção de tecnologias no setor público. Por essa razão, entende-se que a adoção do SEI no âmbito público ainda é tema que necessita de ampliação do número de estudos neste país.

\section{Referências bibliográficas}

AGARWAL R.; PRASAD J. Are individual differences germane to the acceptance of new information technologies? Decision Sciences, v. 30, n. 2, p. 361-391, 1999.

A conceptual and operational definition of personal innovativeness in the domain of information technology. Information Systems Research, v. 9, n. 2, p. 204215, 1998.

The role of innovation characteristics and perceived voluntariness in the acceptance of information technologies. Decision Sciences, v. 28, n. 3, p. 557-582, 1997.

BOUWMAN, H.; HoOfF, B. van den; WIJNGAERT, L. van de; DICK, J. van. Information and communication technology in organizations. Londres: Sage, 2005.

BRASIL. Decreto no 8.539, de 8 de outubro de 2015. Dispõe sobre o uso do meio eletrônico para a realização do processo administrativo no âmbito dos órgãos e das entidades da administração pública federal. 2015. Disponível em: < http://www. planalto.gov.br/ccivil_03/_Ato2015-2018/2015/Decreto/D8539.htm>. Acesso em: 23/08/2018.

CHURCHILL, G. A. Jr. A paradigm for developing better measures of marketing constructs. Journal of Marketing Research, v. 16, p. 64-73, 1979.

Colville, I.; CARTER, M. Innovation as the practice of change in the public sector. In: OSBORNE, S.; BRoWN, L. (Eds.). Handbook of innovation in public services. Cheltenham, UK: Mpg Books Group, 2013. p. 91-106.

DEUS, L.; FARIAS, J. A adoção do processo eletrônico de controle externo (E-TCU) no Tribunal de Contas da União: a experiência dos gestores envolvidos. Revista de Administração e Inovação, v. 12, n. 3, p. 269-291, 2015. 
Dos SANTOS, A. M. Fatores influenciadores da adoção e infusão de inovações em TI. In: SIMPÓSIO DE EXCELÊNCIA EM GESTÃO E TECNOLOGIA, 4., Resende, Pôster. 2007.

EAgly, A.; Chaiken, S. Attitude structure and function. In: GILBERT, D. T.; FISKE, S. T.; LINDZEY, G. (Eds.). The handbook of social psychology. Volume 1, 4. ed. . Boston, MA: McGraw-Hill. 1998. p. 269-322.

EMANI, Srinivas et al. Patient perceptions of a personal health record: a test of the diffusion of innovation model. Journal of medical Internet research, v. 14, n. 6, p. e150, 2012.

Hair JR., J. F.; Anderson, R. E.; TAtham, R. L.; Black, W. C. Análise multivariada de dados. 5. ed. Porto Alegre: Bookman, 2005.

HUNG, S.; HUNG, W.; TSAI, C.; JIANG, S. Critical factors of hospital adoption on CRM system: organizational and information system perspectives. Decision support systems, v. 48, n. 4, p. 592-603, 2010.

Karahanna, E.; Straub, D. W.; Chervany, N. L. Information technology adoption across time: a cross-sectional comparison of pre-adoption and post-adoption beliefs. MIS Quarterly, v. 23, n. 2, p. 183-213, 1999.

KLERING, L. R.; ANDRADE, J. A. Inovação na gestão pública: compreensão do conceito a partir da teoria e da prática. In: JACOBI, P.; PINHO, J. A. Inovação no campo da gestão pública local, novos desafios, novos patamares. Rio de Janeiro: Editora FGV, 2006.

LEAL, E. Fatores determinantes do uso de inovação tecnológica na educação à distância: um estudo com docentes dos cursos na área de negócios. 2012, 141 f. Tese (Doutorado em Administração) - Escola de Administração de Empresas, Fundação Getúlio Vargas, São Paulo. São Paulo, 2012.

LEAL, E. A.; AlBertin, A. L. Construindo uma escala multi-itens para avaliar os fatores determinantes do uso de inovação tecnológica na educação a distância. Revista de Administração e Inovação, v. 12, p. 315-341, 2015.

LEE, N.; HOOLEY, G. The evolution of "classical mythology" within marketing measure development. European Journal of Marketing, v. 39, n. 3/4, p. 365-385, 2005.

LU, Y.; DENG, Z.; WANG, B. Exploring factors affecting Chinese consumers' usage of short message service for personal communication. Information Systems Journal, v. 20, n. 2, p. 183-208, 2010.

Ministério do Planejamento, Desenvolvimento e Gestão. Processo Eletrônico Nacional (PEN) - Sistema Eletrônico de Informações (SEI). 2018. Recuperado de: $\quad<$ http://www.planejamento.gov.br/pensei/adesao-ao-processo-eletroniconacional-pen>. Acesso em 23/08/2018.

Miranda, M. Q.; Farias, J. S.; SCHWARTZ, C. A.; AlmeIDA, J. P. L. Technology adoption in diffusion of innovations perspective: introduction of an ERP system in a non-profit organization. Revista de Administração e Inovação (RAI), v.13, n. 1, p. 48-57, 2016.

MOORE, G. C.; BENBASAT, I. Development of an instrument to measure the perceptions of adopting an information technology innovation. Information Systems Research, v. 2, p. 192-222, 1991.

PAsquALI, L. Análise Fatorial para pesquisadores. Brasília: LabPAM Saber e Tecnologia, 2012. 267 p. 
Perez, G.; ZWicker, R.; Zilber, M. A.; Medeiros JR., A. Adoção de inovações tecnológicas na área de saúde: um estudo sobre sistemas de informação sob a ótica da teoria de difusão. Revista de Gestão da Tecnologia e Sistemas de Informação, v. 7, n. 1, p. 71-94, 2010.

PINSKY, D. O uso do livro eletrônico no ensino superior sob a ótica dos professores universitários e profissionais de editoras. 2009. 141 f. Dissertação (Mestrado em Administração) - Faculdade de economia, Administração e Contabilidade, Universidade de São Paulo, São Paulo, 2009.

Plouffe, C. R.; Hulland, J. S.; VANDENBOSCH, M. Research report: richness versus parsimony in modeling technology adoption decisions - understanding merchant adoption of a smart card-based payment system. Information Systems Research, v. 12, n. 2, p. 208-222, 2001.

RIBEIRO Neto, L. G. Os impactos da tecnologia de informação nas organizações: uma visão política. R. Un. Alfenas, Alfenas, v. 5, p. 95-101, 1999.

RODRIGUES, S. B. A informática na organização e no trabalho. Revista de Administração de Empresas, São Paulo, v. 3, n. 29, p. 43-50, 1988.

Rogers, E. M. Diffusion of innovations. New York: The free press, 1983.

TORNATZKY, L.; FLEISCHER, M. The process of technology innovation. Lexington: Lexington Books, 1990.

VENKATESH, V.; BALA H. Technology acceptance model 3 and a research agenda on interventions. Decision sciences, v. 39, n. 2, p. 273-315, 2008.

VENKATESH, V.; MORRIS, M. G.; DAVIS, G. B.; DAVIS, F. D. User acceptance of information technology: toward a unified view. MIS Quarterly, v. 27, n. 3, p. 425-478, 2003.

Venkatesh, V.; Thong, J.; XU, X. Consumer acceptance and use of information Technology: Extending the unified theory of acceptance and use of technology. MIS Quarterly, v. 36, n. 1, p.157-178, 2012.

Venkatesh, V.; SYKes, T. A.; Venkatraman, S. Understanding e-Government portal use in rural India: role of demographic and personality characteristics. Journal of Information Systems, v. 24, n. 3, p. 249-269, 2014.

Wattal, S.; Racherla, P.; MadviWalla, M. Network Externalities and Technology Use: A Quantitative Analysis of Intraorganizational Blogs. Journal of Management Information Systems, v. 27, n. 1, p. 145-173, 2010.

\author{
Denise de Carvalho Pires Fulton \\ Universidade de Brasília (UnB). Contato: denisecpl@gmail.com \\ Josivania Silva Farias \\ Universidade de Brasília (UnB). Contato: josivania.mkt@gmail.com \\ Solange Alfinito \\ Universidade de Brasília (UnB). Contato: solange.alfinito@gmail.com \\ Juliana Pascualote Lemos de Almeida \\ Universidade de Brasília (UnB). Contato: juliana.pascualote@gmail.com
}

